\title{
Coping Complexity Model: Coping Stressors, Coping Influencing Factors, and Coping Responses
}

\author{
Kathleen Hudson \\ University of Texas at Tyler, Tyler, TX, USA \\ Email: khudson@uttyler.edu
}

Received 14 January 2016; accepted 13 March 2016; published 16 March 2016

Copyright (C) 2016 by author and Scientific Research Publishing Inc.

This work is licensed under the Creative Commons Attribution International License (CC BY). http://creativecommons.org/licenses/by/4.0/ c) (i) Open Access

\begin{abstract}
Aim: To describe the complex phenomena of coping involving the stressors, influencing factors, and responses of coping via a mid-range theory Background: Previous models have presented the concepts of coping, this model expands previous models, creates a revision which is more complex and more comprehensive than previous work. Design: Model includes components of initial stressors, key critical coping influencing factors, and various types of coping responses, either healthy or unhealthy responses. Results: Coping is complex and multifaceted. This model portrays a comprehensive overview of the facets of coping, including temporary, effective, and destructive coping responses. A list of key influencing factors is presented to assist with highlighting possible factors that can influence the overall potential adaptive (healthy) or maladaptive responses (unhealthy). This model outlines the various possible pathways of coping. Conclusion: This model provides a comprehensive complex coping overview which can be used for education, clinical, and research applications.
\end{abstract}

\section{Keywords}

Coping, Coping Responses, Temporary Coping, Effective Coping, Dysfunctional Coping, Maladaptive Coping, Destructive Coping, Resilience, Hardiness, Thriving, Optimization

\section{Introduction}

Coping is a complex phenomenon with great impact on both individuals and the general community. The more we can understand a person's coping styles and variations when they face stressors, the better we may be able to position the coping style toward more positive outcomes. This presentation of Coping Complexity Model clari- 
fies the coping concepts and relationships while outlining overall coping processes. Initial input stressors are appraised in relation to their threat or challenge. Numerous coping influences impact the type of coping that occurs. Subsequently, the coping response occurs with three main potential coping responses described. These responses include: temporary, effective (functional, adaptive, and integrative), along with destructive (dysfunctional/maladaptive) coping. Each coping outcome has a different impact for the individual, group, organization and/or culture involved. A general evaluation of this model is presented. This model can be used for increasing your understanding of coping's complexities, and also, be assistive in research into the complex facets of stress and stress' coping responses.

\section{Background}

What are optimal responses to the various stressors when attempting to build a thriving and resilient personality and stronger relationship within and between various environments? Can an existence where the stressors and the coping competencies are more in balance be created? The concept of coping is quite complex and has both adaptive and destructive facets. This coping model details various key stressors and the subsequent types of coping responses with resultant outcomes. To be well balanced, a diverse range of effective coping skills must be present. Additionally, the availability of resources and supports must be available to assist as daily challenges appear. When stressors are faced, a better prepared and (positively) experienced person can maximize their reach for full potential and constructively respond to their various stressors.

In moving toward a better understanding of the complexity of coping, we need to first understand the complexity of stress and stressors. And second, what are the main coping responses to stress known today? A review of the literature reveals coping theories have been fragmented, within macro and micro socio-cultural, gender, and economic applications. This is due to original works being created within a mechanistic, empiricist and positivist view of health and illness as the dominant western paradigm (Donnelly \& Long, 2003). Various disciplines besides nursing have investigated coping, these include psychology, sociology, medicine and education. Today, holistic, qualitative, and more comprehensive interpretive approaches toward managing stressors and coping are still evolving. This transition is driven by the broader understanding of coping's complex nature and the influences involved within the usual aspects of daily life.

The early work by Selye (1974, p. 27) identified stress as "a nonspecific body response to any demand place upon it”. This stimulated further interest in stress, identifying key stressors and coping. Coping was then defined by Lazarus and Folkman, (1984, p. 141) as "constantly changing cognitive and behavioral efforts to manage specific external and/or internal demands that are appraised as taxing or exceeding the resources of the person." Coping is a process involving an initial appraisal that an event or situation is a threat with a potential impact on one's well-being. Lazarus and Folkman (1984), as early leaders in coping research, described personal perception termed "appraisal" along with susceptibility and threats to wellbeing, along with problem-focused and emotional-focused responses as aspects of stress and coping. Their psychological theory of appraisal, stress and coping were presented as the Transactional Model of Stress and Coping (Lazarus \& Folkman, 1984). Folkman (1997) later revised the model to include event outcomes and negative feedback loops. Coping was defined as managing stressors (internal and external threats) via cognitive and behavioral efforts; manifested in actions, emotions, and thinking responses which can be adaptive or maladaptive. "Within the dominant discourse, stress is naturalized, normalized, problematized, and individualized... with similar views between the lay public and healthcare professionals (Donnelly \& Long, 2003).” Due to the diverse array of health issues investigated in relation to stress and coping, a diverse range of evidence identifies the influencing variables used for the mechanisms of coping.

Within a given situation, decisions or potential response options consider details such as: the overall capacity to respond, influences of previous experience, and related current thought and affect properties. Coping responses are potentially cognitive, emotional, and/or behavioral (Macy, 2007). In the coping process, healthy and constructive coping are desirable as goals. When fostering healthy and constructive coping, more comprehensive understanding is achieved when knowledge interrelated across disciplines and sciences is incorporated into an integrated picture. Theoretical models that "creatively incorporate relevant science about persons or populations of interest into parsimonious concepts and principles that appropriately blends levels of analysis and explicate complex relationships are integrative science (Walker, 1999: p. 98)”. Nilsson (2007, p. 10) describes the unique differences seen between individuals as explanatory for why some respond to a stressor as a threat and another 
would respond perceiving the situation as a challenge. Current coping models must demonstrate this assimilation of many relationships into a broader schema. They must also include a reflection of the social, cultural and economic aspects that define and perpetuate the "stressors" experienced as defined by the worldview in which the stressor is occurring.

Proactive coping efforts seek to modify events before stressors occur and require different skills than reactive coping (Bode, de Ridder, \& Bensing, 2006). When stressors are viewed from the perspective of being challenges a positive affect is elicited. In this context, proactivity and stressors viewed as holistic challenges require the challenging of traditional illness-wellness perspectives on stress. When working toward aligning stressors with healthy coping responses, the environmental, social, and cultural influences of that experience must be taken into account. When seeking the most constructive responses, developing positive skills to quickly determine a comprehensive evaluation of the issue and potential solutions are warranted. It is important to resist the dominant paradigms' efforts to deal with specific problems in a fractionalized "illness" manner. Instead, it is reasonable to step back and focus on integrated and holistic approaches toward stress management, and therefore produce constructive coping.

Today's research continues to evolve toward more comprehensive evidence on how to best develop a coordinated screening, education, and ongoing support in facilitating a maximized level of healthy coping. There is no one simple method for establishing an initial understanding and then successful coping with stress-due to the unique experiences of stress being interwoven into the complex fabric of everyday life. A coping model which highlights the various intricacies of coping responses is outlined below. This model can facilitate a more in-depth understanding of coping's complexity.

\section{Schematic and Model: Coping Complexity Model}

The Coping Complexity Model depicts the stressors, coping processes, and subsequently, the various responses that are possible. The coping process is initiated by a stressor(s). Stress is defined and influenced by social, cultural, cognitive and environmental norms and values which impact the entire coping experience. The stress experience can present at an individual, group, community, or cultural level. It is also influenced by the timing, number and nature (level or significance) of the stressor(s) and the specific personality traits involved. This heuristic coping model, Coping Complexity Model, and its influencing factors are presented in Figure 1, with the concepts clarified below.

\section{Key Concepts}

\subsection{Coping Complexity}

Integrative coping is used here to represent the "understanding of complex health issues which transcend disciplinary and knowledge boundaries and provide opportunities to view phenomena from diverse perspectives" (King \& Hegadoren, 2006). This integrative framework exists because of the diversity in stressor types, each individual's personality/emotional make up, and the potential ranges and degrees of responses that result to create an outcome. For example, gender intimately influences stressors and coping responses (Krishnakumar, Narine, Soonthorndhada, \& Thianlai, 2015). Also, one’s self-esteem and emotional intelligence can mediate stressors and stress responses (Jesse, Kim, \& Herndon, 2014; Park, Ha, Lee, \& Lee, 2014).

This integration aligns with systems theory which portrays a perspective of integrated, interdependent and interactive parts in addition to feedback loops as the foundation of the individual, group, organization, and culture. Integrative coping is replaced by the new term coping complexity due to the current concept revision, which includes refinement of the overall concept and specific details, as outlined in Figure 1. The Coping Influence Table outlines factors that influence the situation and involves various dynamics including sociocultural, emotional, cognitive, and physical aspects.

\subsection{Heuristic}

The term heuristic refers to "involving or serving as an aid to learning, discovery, or problem-solving by experimental and especially trial-and-error methods, and of or relating to exploratory problem-solving techniques that utilize self-educating techniques to improve performance” (Merriam-Webster Online Dictionary, 2009). Heuristic applied here relates to using a diverse range of coping styles and strategies that will align with each 


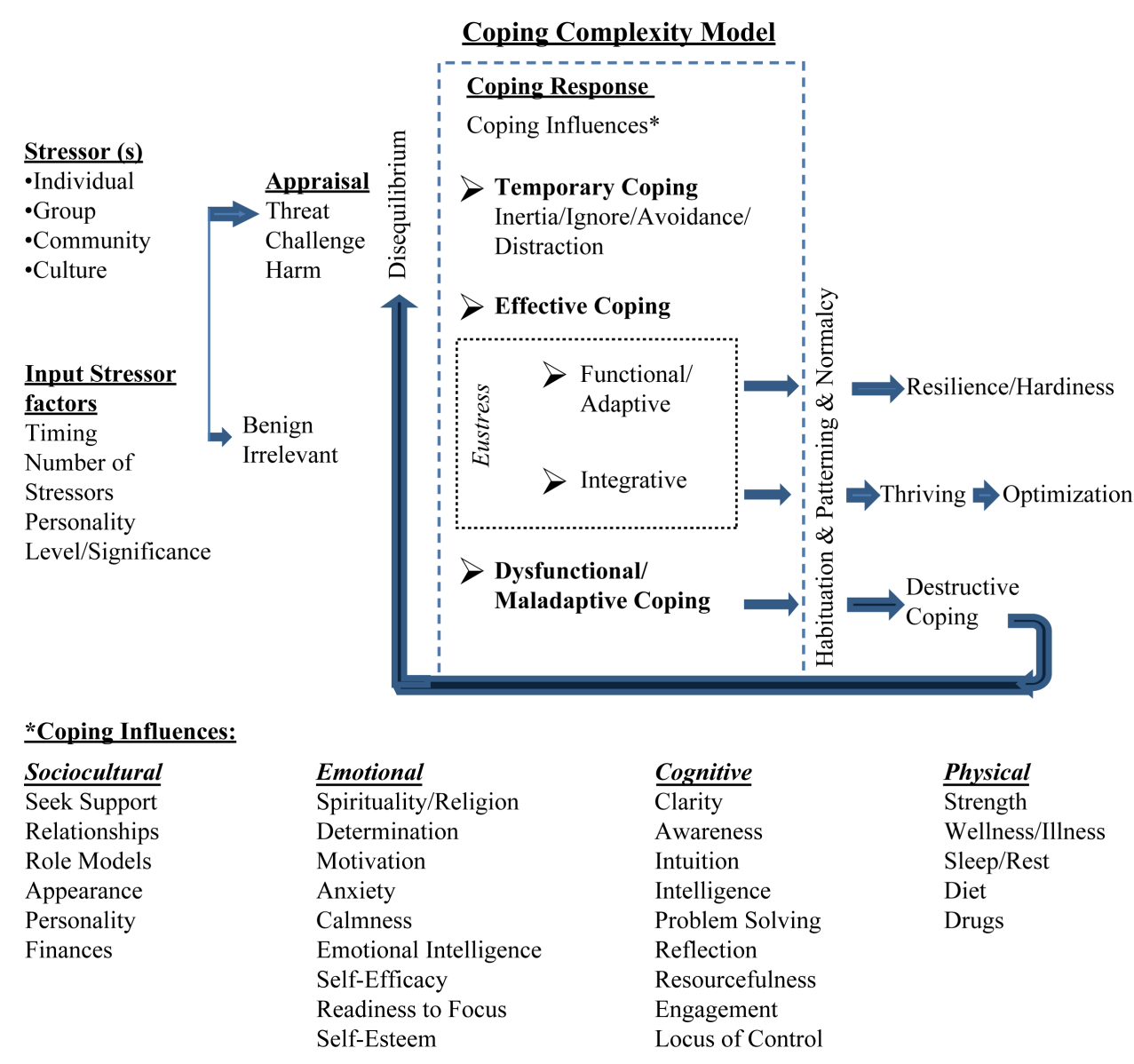

Figure 1. Coping complexity model.

specific stressor issue and contextualized situation being dealt with at that unique time. Heuristic coping exists within this integrative and complex framework. Because current experiences of stressors and impacts are unique for each individual or situation and built upon that person's previous unique experiences, an informed (or not informed) "trial and error" response for that given situation is exhibited in an effort to problem-solve that specific situation.

\subsection{Coping}

Coping is defined as, "constantly changing cognitive and behavioral efforts to manage specific external and/or internal demands that are appraised as taxing or exceeding the resources of the person" (Lazarus \& Folkman, 1984: p. 141). Coping has many layers. Coping begins with influences of readiness to focus and engagement. Coping can be short-term yet assistive, termed here as temporary. It can also be positive, referred to as functional and integrative coping. Or, it can be non-assistive, identified here as dysfunctional and maladaptive coping. This leads to negative outcomes and destructive situations for the person, group, organization, and culture.

\subsection{Stress}

"Stress is the nonspecific response of the body to any demand made upon it (Selye, 1974: p. 27)." Stresses can be internal or external in origin. Four levels of stress/coping exist: within an individual, group, organization, and culture (Ekedahl \& Wengstrom, 2008).

\subsection{Appraisal/Disequilibrium}

Appraisal is the individual's perception or assessment of the stressor and influences how one will cope (or re- 
spond) with the stressful event (LeSergent \& Haney, 2005). Identifying and deciphering the stressor begins during appraisal. The appraisal determines if the stressor is considered a threat or challenge. This is followed by a response which can be benign and irrelevant, helpful and productive, or it can be harmful and destructive. If the stressor is perceived as requiring a response then disequilibrium occurs. This disequilibrium leads to a dealing withor coping response.

\subsection{Disequilibrium}

Disequilibrium is a sensation of disharmony as a result of exposure to threatening stressors. Within the stress response process, disequilibrium is an uncomfortable psychological space and the individual will make efforts to move to a more comfortable state via one of the coping responses.

\subsection{Temporary Coping}

Temporary coping relates to using an interim strategy to deal with stressors in that the stressors have not been dealt with in a permanent or resolving fashion. The issue(s) will need to be addressed again at some point in the near future. A reprieve from deciding what to do is currently present. This involves a limited-time response such as inertia, ignoring, avoidance or distraction so as not to experience the uncomfortable stress-related disequilibrium.

\subsection{Effective Coping}

"Effective coping" relates to the response when an individual is coping with current stressors with their "head just above the water". They are functioning at a safe and reasonable level. Their coping would be considered fragile. Another individual who is exposed to repeated intense stressors and then deals with each stressor adequately would be considered to be effectively coping. Some aspects of spirituality or religion can play major roles in the perception and reaction to stressors and lead to effective coping (Kuo, Arnold, \& Rodriquez-Rubio, 2014). During these interactions, each stressor is separated by enough time to fully recover, before the next stressor occurs. Having effective problem solving strategies (developed over time) can assist with effective coping responses (Al-Zayyat \& Al-Gamal, 2013).

\subsection{Eustress}

Eustress is defined as a positive response to a stressor, as indicated by the presence of positive psychological states (Simmons \& Nelson, 2001). Lazarus and Folkman (1984) view psychological states as multidimensional, this alludes to being able to simultaneously have more than one emotional association related to a stressor/challenge at a time. Eustress is desirable as a means toward healthier problem-solving.

\subsection{Functional/Adaptive Coping}

Adaptive/Functional Coping is adjusting in a positive and constructive way to the stressors that are encountered. One good outcome of repeated adaptive coping is the development of resilience and hardiness traits. Resilience results from repetitive use of adaptive coping and maintains the wellbeing of the individual at a functional level within their current internal and external environments (Gillespie, Chaboyer, \& Wallis, 2007). Resilience may derive from physiological or psychological coping processes rather than external risk or protective factors (Rutter, 2006). Functional and adaptive coping describe a healthful response to various stressors that are faced.

\subsection{Integrative Coping}

Integrative coping incorporates the complex, interdependent and interactive systems of socio-cultural, emotional, cognitive, and physical aspects at the highest level of integration to result in the best possible outcomes. "Our understanding of stress would be incomplete if considered in one tradition in isolation from another, requiring all biological (physiological and immunological), psychological (emotional and perceptual), and cognitive aspects involved (King \& Hegadoren, 2006).” Within an integrative analysis, an individual's (or group's, organization's, society's or culture's) productive and successful response to stressors can enhance their overall wellbeing by making the situation improved in an overall positive and constructive way. Over repeated situations 
with resultant high levels of success, this develops into resilience (Sattler, Boyd, \& Kirsch, 2014). Resilience can lead to thriving. Thriving is actual, significant, positive progression and growth that occurs because of constructively dealing with stress and benefiting from the lessons learned within these positive experiences. Thriving is a higher form of successful coping. Even higher forms of successful thriving involve optimization. Optimization involves promotion of the best goals and high standards (Clancy, 2011; Penningroth \& Scott, 2012).

\subsection{Dysfunctional/Maladaptive and Destructive Coping}

Destructive coping occurs as a result of dysfunctional and maladaptive responses to stressors. Destructive coping perpetuates harm to the individual, group, organization, society or culture. These responses are destructive to physical and psychological wellbeing. Examples of this type of coping responses are: substance abuse, excessive, repetitive anger and hostility, aggression toward others, and more in-depth levels of depression (Sullivan, Flanagan, Dudley, Holt, Mazure, \& McKee, 2015). This form of coping can become quite debilitating. Both destructive and temporary coping require revisiting and addressing the initiating stressor(s) to transition to a more constructive form of dealing with and alleviating the stressors.

\subsection{Habituation and Patterning}

Habituation "refers to an organism's loss of responsiveness to a repeated or continuously present stimulus (Greenwood, 1993)." Patterning is the redundancies of similar responses that may evolve over time due to the repeated need to respond to stressors and stressful situations. Both habituation and patterning of responses can result as coping responses repeat over time from similar, and recurrent events/situations.

\subsection{Normalcy}

Normalcy is a state returned to or efforts made toward reestablishing a situation as close to where the person was in the first place, before the stressors. Normalcy involves making adjustments or adapting to the situation to create equilibrium for the individual or situation, "to adapt to the stressful experience to maintain a sense of normalcy (Selye, 1974).” Repeated stress responses create a sense or even a destructive sense of one’s normalcy.

\subsection{Coping Influences}

Coping Influences shape the nature of responses to stressors. Research continues to investigate the factors influencing the complex processes of coping. The coping influences identified here transverse four domains including socio-cultural, emotional, cognitive and physical. Figure 1, the Coping Complexity Model, highlights the key factors impacting perceptions and reactions to stressors.

The assumptions of the Coping Complexity Model and underlying theory are: 1) Stress is an inevitable experience of life (Existence statement); 2) The appraisal of a stressor as potentially harmful leads to disequilibrium (Relational statement); 3) Coping is the natural tendency for all human beings faced with any disequilibrium situation (Relational statement); 4) Coping responses vary, including temporary, functional/adaptive, integrative, and dysfunctional/maladaptive coping and occur on sociocultural, emotional, cognitive and physical levels (Existence statement); and 5) Influencing interactions can influence the form of coping responses utilized (Relational statement). Additional statements that exist involving the Coping Complexity Model are:

1) A stressor precedes and then elicits an appraisal. Accurately appraised benign or irrelevant stressors are dismissed. If the stress, threat or challenge is something that can create an impact, then a stress response is required. Many responses are available and various responses can occur.

2) Coping response levels vary depending on the perception and the actuality of the threat(s), perception and resources to respond, personality traits and many other factors (see Figure 1. Coping Influences Table).

3) Responses to stressors can be constructive when using the responses of just coping, or functional and integrative strategies. Response to stressors can be destructive when using dysfunctional/maladaptive coping.Both temporary coping and destructive coping require a revisiting of the underlying issue to move toward a constructive resolution.

The Coping Complexity Model presents a framework to assist with understanding the complex phenomena of coping as it is known and exists today. Dudley-Brown (1997, p. 80) supports new models having a "need for consistency with the present reality in nursing, the overall current philosophical or culture of nursing where it 
would be used or applied.” Based on this model, the three following propositions may be helpful in progressing further exploration:

1) Humans experience stress, disequilibrium, and then react with various coping responses. A more thorough understanding of coping and the influences of coping can guide insight during disequilibrium decision-making,

2) When factors influencing potential choices are known, an individual, group, community, society or culture can make wiser and possibly healthier coping decisions, and

3) Humans can become efficient at analyzing stressors and potential responses and determine their most healthy and beneficial response.

\section{Coping Complexity Model Evaluation}

This Coping Complexity Model includes relevant concepts from the literature involved in the complex, multifaceted coping process. Additional components are added as noted in the "temporary coping" designation. The temporary and destructive coping feedback leads back to an uncomfortable sense of disequilibrium. The "functional/adaptive" coping can, over time, develop an outcome of "resilience/hardiness", along with the same transition for "integrative" coping leading to the outcome of "thriving". "Eustress" is added to incorporate this concept into the model as a psychological antecedent to adaptation and integrative coping. These linked central concepts can help identify and clarify relationships with both short and long term focuses.

Coping complexity components are highlighted and reflected in the list of the influencing factors. Each factor listed can influence decision making as an individual or group is reviewing their "stressful" situation. Each factor is separate, however, these factors inter-influence at the same time to varying degrees when one experiences multiple stressors. For example, a severe lack of sleep can impact the level of problem solving, motivation, and one's relationships. An understanding of coping influences can foster constructive, strong and reliable habits and patterning which can assist to make future decision making healthier, clearer and more effective. The usefulness of the model relies on its comprehensiveness in comparison to previous models, and its ease to scan and understand.

\subsection{Generalizability}

Stress is a common and usual life experience. Any one individual or group, community, society and culture can utilize any or all of these coping responses at any given time, depending on the situation and the interacting coping influences. This model identifies usual key coping responses which are readily observed in everyday life. Thus, this model is easy to assimilate, apply and use. By representing a moderate level of abstraction, this level provides flexibility in being able to be applied across various contexts. Within these various contexts, the model may assist to guide and set goals toward more constructive resolution from the triggering stressors. Specifically, it may be help to highlight where improvements can be focused by identifying where decisions and/or behaviors are destructive and not beneficial. In reevaluating the potential responses, the responses can be modified and adjustments made so they can be more constructive. Just like in life, negative decisions can be made when, previously, positive ones have occurred. And, positive decisions can be made, when, previously negative ones have occurred. Thus stress responses can potentially be bidirectional, as influenced from the current stressors, as toward positive or negative outcomes. It is important to highlight that thriving is achievable over time with repeated high quality coping. In situations where effective responses have been made, these responses can be researched and similar responses replicated in other contexts to encourage the overall benefits of effective coping and thriving over time.

This model supports an individual's decision making by providing an informed flexibility in that a person has a framework to "decide from" in relation to stressors and how to respond, whether constructive or destructive. Making better decisions with higher quality responses is desirable in all interactions. This is applicable and relevant to four areas of usefulness as identified by Meleis (1985) including practice, education, research, and administration.

\subsection{Parsimony}

According to Houser (2012, p. 456), parsimony means, "reduced to the fewest components, a parsimonious model is the simplest one that will demonstrate a concept." Houser (2012, p. 456) follows with, "a parsimonious 
explanation is one that is the most focused while providing the best overview of the final conclusions.” This model uses one or two clear terms to represent each concept. The relationships are outlined in the process flow that is exhibited in the decision making process. Both favorable constructive and unfavorable destructive coping responses are represented.

\subsection{Testability}

This Coping Complexity Model provides direction for further coping investigation and development. Aspects of this revised theory have yet to be tested. For example, research could clarify the best means to facilitate student development of specific resilience and thriving characteristics within the classroom and external settings. In addition, research evidence to determine the best means to lessen stress and create eustress would be beneficial. Also, it would be important to determine which specific coping strategies or methods produce the longevity of best and highest level of coping. Another important question is "what is the most efficient means to turn around maladaptive coping behaviors to foster a transition toward thriving?” Thus many questions can be generated from this model's application.

Instruments to investigate coping are available (Schwarzer \& Schwarzer, 1996), and continue to evolve. King and Hegadoren (2006) describe emotional, cognitive and physiological research approaches contributing to understanding stress and possible ways to negate its impact. They discuss issues surrounding a participants' subjective interpretation and evaluation when measuring the cognitive processes associated with the stressful situations (in addition to the dynamic nature of moods and coping strategies). Interventions can be empirically investigated using this framework to identify both effective and ineffective coping strategies. The strategies used could then be targeted toward healthier coping methods.

The Coping Complexity Model can be used for both qualitative and quantitative research questions. It can provide a framework to guide a study's intervention selection when researching coping variances. Existing instruments for investigating coping are listed as described in Schwarzer and Schwarzer (1996). Issues with Instruments according to this article are 1) how the tools have been generated, some with theory, and some with empirical foundations, and a balance between the two is not present; 2) studying inter-individual variance or studying intra-individual variances is another question when researching the complexity of coping; 3) using real-life versus hypothetical scenarios complicates the analysis as there are pros and cons for each; and 4) analysis of the multidimensionality involved in coping because it can easily complicate a study's analysis of results.

This process orientation instead of a cook-book approach (one size fits all) is realistic and reflects a truer lived experience reflection. The influencing factors can provide insight into addressing the underlying reasons for the occurrence of specific coping decisions. This model creates a structure for education by providing the basic outline for potential responses. It is realistic and humanistic in that it reflects both positive and negative aspects of coping, supporting self-determination and ownership of difficult decision making. Difficult decisions are common within many contexts. If the framework assists to provide clarity perspectives during difficult decision making and prioritizing then it is useful.

\subsection{Strengths and Weaknesses}

The strength of this Coping Complexity Model is in its transparency, open cultural framework, comprehensiveness, simplicity and clarity. Coping well requires expertise developed primarily from experience, interacting with constructive others or mentor(s) during the stressors, which assists to develop positive perspectives toward problem solving. Coping is a complex phenomenon and involves various processes be described to ensure a general understanding of this current model. Further research and refinement of the structure and processes within this model are necessary to move forward in stress management and coping care. The model's weaknesses lie in that it is yet to be empirically tested. Refinement of relationship details between concepts still requires further research and enhancement.

\section{Conclusion}

This Coping Complexity Model consolidates current coping knowledge and delineates various coping responses. Coping has both potential adaptive and maladaptive responses. Within the context of coping, fostering constructive coping (and potentially enhancing the transformation to thriving) within the environment would be a won- 
derful asset to bring forth. This model requires empirical testing and refinement to assist toward fostering these efforts. Coping occurs every day at to various degrees and with various responses. Depending on one's experience, exposure, and reflection, the actual responses can be modified so as to create a healthier pattern toward positive coping strategies and results.

\section{References}

Al-Zayyat, A. S., \& Al-Gamal, E. (2013). Perceived Stress and Coping Strategies among Jordanian Nursing Students during Clinical Practice in Psychiatric/Mental Health Courses. International Journal of Mental Health, 23, 326-335. http://dx.doi.org/10.1111/inm.12054

Bode, C., de Ridder, D. T. D., \& Bensing, J. M. (2006). Preparing for Aging: Development, Feasibility, and Preliminary Results of an Educational Program for Midlife and Older Based on Proactive Coping Theory. Patient Education and Counseling, 61, 272-278. http://dx.doi.org/10.1016/j.pec.2005.04.006

Clancy, T. R. (2011). Improving Processes through Evolutionary Optimization. Journal of Nursing Administration, 41, 340342.

Donnelly, T. T., \& Long, B. C. (2003). Stress Discourse and Western Biomedical Ideology: Rewriting Stress. Issues in Mental Health Nursing, 24, 397-408. http://dx.doi.org/10.1080/01612840305316

Dudley-Brown, S. L. (1997). The Evaluation of Nursing Theory: A Method for Our Madness. International Journal of Nursing Studies, 34, 76-83. http://dx.doi.org/10.1016/S0020-7489(96)00024-7

Ekedahl, M., \& Wengstrom, Y. (2008). Coping Processes in a Multidisciplinary Healthcare Team—A Comparison of Nurses In Cancer Care and Hospital Chaplains. European Journal of Cancer Care, 17, 42-48.

http://dx.doi.org/10.1111/j.1365-2354.2007.00801.x

Folkman, S. (1997). Positive Psychological States and Coping with Severe Stress. Social Science \& Medicine, 45, $1207-21$. http://dx.doi.org/10.1016/S0277-9536(97)00040-3

Gillespie, B. M., Chaboyer, W., \& Wallis, M. (2007). Development of a Theoretically Derived Model of Resilience through Concept Analysis. Contemporary Nurse, 25, 124-135. http://dx.doi.org/10.5172/conu.2007.25.1-2.124

Greenwood, J. (1993). The Apparent Desensitization of Student Nurses during Their Professional Socialization: A Cognitive Perspective. Journal of Advanced Nursing, 18, 1471-1479. http://dx.doi.org/10.1046/j.1365-2648.1993.18091471.x

Houser, J. (2012). Nursing Research: Reading, Using, and Creating Evidence. Nursing Research (2nd ed.). Sudbury, MA: Jones \& Bartlett Learning, Inc.

Jesse, E., Kim, H., \& Herndon, C. (2014). Social Support and Self-Esteem as Mediators between Stress and Antepartum Depressive Symptoms in Rural Pregnant Women. Research in Nursing \& Health, 37, 241-252. http://dx.doi.org/10.1002/nur.21600

King, S. L., \& Hegadoren, K. M. (2006). An Integrative Science Approach: Value Added in Stress Research. Nursing and Health Sciences, 8, 114-119. http://dx.doi.org/10.1111/j.1442-2018.2006.00256.x

Krishnakumar, A., Narine, L., Soonthorndhada, A., \& Thianlai, K. (2015). Family Stressors, Home Demands and Responsibilities, Coping Resources, Social Connectedness, and Thai Older Adult Health Problems: Examining Gender Variations. Journal of Aging and Health, 27, 257-283. http://dx.doi.org/10.1177/0898264314549658

Kuo, B. C. H., Arnold, R., \& Rodriquez-Rubio, B. (2014). Mediating Effects of Coping in the Link between Spirituality and Psychological Distress in a Culturally Diverse Undergraduate Sample. Mental Health, Religion, \& Culture, 17, 173-184. http://dx.doi.org/10.1080/13674676.2013.780015

Lazarus, R., \& Folkman, S. (1984). Stress, Appraisal and Coping. New York: Springer.

LeSergent, C. M., \& Haney, C. J. (2005). Rural Hospital Nurse’s Stressors and Coping Strategies: A Survey. International Journal of Nursing Studies, 42, 315-324. http://dx.doi.org/10.1016/j.ijnurstu.2004.06.017

Macy, R. J. (2007). A Coping Theory Framework toward Preventing Sexual Revictimization. Aggression \& Violent Behavior, 12, 177-192. http://dx.doi.org/10.1016/j.avb.2006.09.002

Meleis, A. I. (1985). Theoretical Nursing: Development and Progress. Philadelphia, PA: J. B. Lippincott Co.

Merriam-Webster Online Dictionary. Heuristic. Retrieved 4 April 2009. http://www.merriam-webster.com/dictionary/heuristic

Nilsson, D. (2007). Adapting Coping Theory to Explain the Concept of Adjustment. Social Work in Health Care, 45, 1-20. http://dx.doi.org/10.1300/J010v45n02 01

Park, H.-S., Ha, J.-H., Lee, M.-H., \& Lee, H.-J. (2014). The Relationship between Emotional Intelligence and Stress Coping of Nurses. Journal of Korean Academy of Fundamentals of Nursing, 21, 466-474.

http://dx.doi.org/10.7739/jkafn.2014.21.4.466 
Penningroth, S. L., \& Scott, W. D. (2012). Age-Related Differences in Goals: Testing Predictions from Selection, Optimization, and Compensation Theory and Socioemotional Selectivity Theory. International Journal of Aging \& Human Development, 74, 87-111. http://dx.doi.org/10.2190/AG.74.2.a

Rutter, M. (2006). Implications of Resilience Concepts for Scientific Understanding. Annals of the New York Academy of Sciences, 1094, 1-12. http://dx.doi.org/10.1196/annals.1376.002

Sattler, D. N., Boyd, B., \& Kirsch, J. (2014). Trauma-Exposed Firefighters: Relationships among Posttraumatic Growth, Posttraumatic Stress, Resource Availability, Coping and Critical Incident Stress Debriefing Experience. Stress \& Health, 30, 356-365. http://dx.doi.org/10.1002/smi.2608

Schwarzer, R., \& Schwarzer, C. (1996). A Critical Survey of Coping Instruments. In M. Zeider, \& N. S. Endler (Eds.), Handbook of Coping (pp. 107-132). New York: Wiley.

Selye, H. (1974). Stress without Distress. New York: Lippincott.

Simmons, B. L., \& Nelson, D. L. (2001). Eustress at Work: The Relationship between Hope and Health in Hospital Nurses. Health Care Management Review, 26, 7-18. http://dx.doi.org/10.1097/00004010-200110000-00002

Sullivan, T. P., Flanagan, J. C., Dudley, D. N., Holt, L. J., Mazure, C. M., \& McKee, S. A. (2015). Correlates of Smoking Status among Women Experiencing Intimate Partner Violence: Substance Abuse, Posttraumatic Stress, and Coping. The American Journal on Addictions, 24, 546-553. http://dx.doi.org/10.1111/ajad.12261

Walker, L. O. (1999). Is Integrative Science Necessary to Improve Nursing Practice? Western Journal of Nursing Science, 21, 94-102. http://dx.doi.org/10.1177/01939459922043730 